\section{Variants of Chinese Postman Problems and a Way of Solving through Transformation into Vehicle Routing Problems}

\author{
M.K. Gordenko<mgordenko@hse.ru> \\ S.M.Avdoshin<savdoshin@hse.ru> \\ Department of Software Engineering, \\ National Research University Higher School of Economics, \\ 20, Myasnitskaya st., Moscow, 101000 Russia
}

Abstract. In this article, the routing problems are described. It is shown, that almost all routing problem can be transformed into each other. An example of the Mixed Chinese Postman problem is discussed. The article gives an overview of various variants of Chinese Postman Problem. For all problems the mathematical formulation is given. Moreover, the useful reallife application is presented, too. Then, the article provides a table of possible Chinese Postman problems and identifies parameters that can be varied for obtaining new problems. Five parameters have been identified, such as: presence of set of edges; presence of set of arcs; presence of edges with cost, depending on traversing; the presence of set of required edges; the presence of set of required arcs. It was shown that by varying these parameters one can obtain tasks that were not described earlier but can be used in real life. Four new tasks were identified. Then it is shown that the Chinese Postman problem can be solved as another routing tasks through graph transformations. The method for transforming Chinese Postman problem into the Generalized Travelling Salesman problem is given. Then the results of solving the above problem are presented by simple algorithms, and their effectiveness is shown. The research is not over yet. The testing of other algorithms is planned.

Keywords: Generalized Routing Problem; Arc Routing Problem; Chinese Postman Problem; Generalized Travelling Salesman Problem

DOI: $10.15514 /$ ISPRAS-2018-30(3)-16

For citation: Gordenko M.K., Avdoshin S.M. The Variants of Chinese Postman Problems and Way of Solving through Transformation into Vehicle Routing Problems. Trudy ISP RAN/Proc ISP RAS, vol. 30, issue 3, 2018, pp. 221-232. DOI: 10.15514/ISPRAS-2018-30(3)-16

\section{Introduction}

The General Routing Problem (GRP) is a routing problem defined on a graph where a minimum cost tour is to be found and where the route must include visiting certain required vertices and traversing certain required edges [1]. The routing problems are closely related to the logistic and transportation management. From the theoretical point of view, routing problems are mainly related to determining the optimal set of routes in a graph. In practice, the routing problems are not only the tasks of determining optimal set of routes, they are also the tasks of testing robots, the correctness of links in the application menu and operating systems, interactivity usability of web-sites [2]. The Travelling Salesman Problem (TSP) is one of the routing problem consisting in finding a minimal length closed tour that visits each city once. The TSP is one of the most well-known routing problem. Another practical, but less well-known problem is the Chinese Postman Problem (CPP). The CPP is finding a shortest closed path that visits every edge or arc of a graph. The CPP has a simple formulation and a lot of potentially useful applications, but today is poorly understood.

The article gives an overview of various CPPs, provides mathematical formulations of problems, and describes the scope of the problem. In addition, the article cites references to the literature, in which the various ways of transforming different types of ARP to VRP is described. Also, the results of the current research of various algorithms for solving the problem of a Generalized Traveling Salesman Problem (GTSP) are presented.

\section{The Variations of Chinese Postman Problem}

There are a lot of variations of CPP. Below, some of them are described.

\subsection{The Windy Rural Chinese Postman problem}

The Windy Rural Chinese Postman Problem (WRCPP) is a special case of ARP, in which $A_{R} \subseteq A, E_{R} \subseteq E$, and the cost of traversing the edges is depended from the direction of traversing.

WRCPP is a generalization of the CPP in a mixed multigraph. In original CPP problem, it is necessary to find a closed route of minimum length that contains all edges and arcs of the original multigraph at least once. In the real world, it is not always necessary to traverse absolutely all edges and arcs, it is enough to traverse only a certain set of them. Besides, the cost of traversing the edges depend from direction of traversing. The problem of this type is known as the Windy Rural Chinese Postman Problem, which is finding a closed route of minimum length that contains all required edges or arcs of the original multigraph at least once and can contains non-required edges or arcs, so, that the cost of traversing edges depends on traversing direction $[5,6]$.

Fix the edge $\left\{v_{i}, v_{j}\right\}$ (non-oriented pair of vertex) from $E$. Define $\left(v_{i}, v_{j}\right)$ as ordered pair of vertices, meaning the traversing an edge $\left\{v_{i}, v_{j}\right\}$ from vertex $v_{i}$ to $v_{j}$ vertex. Note, that $\left(\exists\left\{v_{i}, v_{j}\right\} \in E\right)\left(C\left(v_{i}, v_{j}\right) \neq C\left(v_{i}, v_{j}\right)\right)$

Let arc be $\left(v_{i}, v_{j}\right) \in A$ ordered pair of vertices, meaning the traversing an $\operatorname{arc}\left(v_{i}, j\right)$ from vertex $v_{i}$ to $v_{j}$ vertex. 
We give a formal formulation of the WRCPP problem, extending it to the case of a mixed multigraph.

Let $I=\left\{1,2, \ldots,\left|E_{R}+A_{R}\right|\right\}, L=\{1,2, \ldots,|V|\}$. On the set of vertices $V$ of $G$ define indexation $\quad \operatorname{inv}=V \rightarrow L,\left(\forall v_{i} \in V\right)\left(\forall v_{j} \in V\right)\left(v_{i} \neq v_{j} \rightarrow i \neq j\right), i=\operatorname{inv}\left(v_{i}\right)$. On the set $E_{R} \cup A_{R}$ of $G$ define indexation inea $=E_{R} \cup A_{R} \rightarrow I$, $\left(\forall u_{i} \in\left(E_{R} \cup A_{R}\right)\right)\left(\forall u_{j} \in\left(E_{R} \cup A_{R}\right)\right)\left(u_{i} \neq u_{j} \rightarrow i \neq j\right), i=\operatorname{inu}\left(u_{i}\right)$.

The solution of WRCPP is the route $\mu=\left(v_{l_{1}}, u_{p_{1}}, v_{l_{2}}, u_{p_{2}}, \ldots, v_{l_{k}}, u_{p_{k}}\right)$, which satisfy for the following [11]:

$u_{p_{i}}=\left\{\begin{array}{l}\left(v_{l_{i}}, v_{l_{i+1}}\right),\left(v_{l_{i}}, v_{l_{i+1}}\right) \in E \\ \left(v_{l_{i}}, v_{l_{i+1}}\right),\left(v_{l_{i}}, v_{l_{i+1}}\right) \in A\end{array} i=1,2 \ldots, k-1\right.$

$u_{p_{k}}=\left\{\begin{array}{l}\left(v_{l_{k}}, v_{l_{1}}\right),\left(v_{l_{k}}, v_{l_{1}}\right) \in E \\ \left(v_{l_{k}}, v_{l_{1}}\right),\left(v_{l_{k}}, v_{l_{1}}\right) \in A\end{array} i=1,2 \ldots, k-1\right.$

$E_{R} \cup A_{R}\left\{u_{p_{1}}, u_{p_{2}}, \ldots, u_{p_{k}}\right\}=\varnothing$

We denote by $C(\mu)=\sum_{i=1}^{k} C\left(u_{p_{i}}\right)$ the cost of traversing the route.

Let $M$ is a set of WRCPP routes. It is needed to find $\mu_{0} \in M$, where

$(\forall \mu \in M)(C(\mu 0) \leq \mathrm{C}(\mu))$

A lot of theoretical and computational works is devoted to WRCPP. WRCPP cannot be solved for polynomial time. In general, the problem of WRCPP is NP-hard [12].

\subsection{The Undirected Rural Chinese Postman problem}

The Undirected Rural Chinese Postman Problem (URCPP) is a particular WRCPP which consists of determining a minimum cost circuit on a graph so that it is possible to traverse a given subset of required edges.

DCPP is a special case of WRCPP, where $A=\emptyset$, and there is not edges, which satisfy (1). So, $\forall\left\{v_{i}, v_{j}\right\} \in E, C\left(v_{i}, v_{j}\right)=C\left(v_{j}, v_{i}\right)$.

The URCPP is known to be an NP-hard problem and it has some interesting real-life applications.

\subsection{The Undirected Chinese Postman problem}

The Chinese Postman problem in the undirected graph (Undirected Chinese Postman Problem, UCPP) is the original statement of the CPP problem, which was firstly introduced by the mathematician Kwang-Mei-Ko in 1960 [2].

UCPP is a special case of WRCPP, where $A=\emptyset, E_{R}=E$ and there is not edges, which satisfy (1). So, $\forall\left\{v_{i}, v_{j}\right\} \in E, C\left(v_{i}, v_{j}\right)=C\left(v_{j}, v_{i}\right)$

If multigraph has Eulerian circuit then this cycle is a solution of UCPP. The algorithm for constructing the Eulerian circuit has $O(|E|)$ time complexity [5].

The Eulerian circuit is existing in an undirected multigraph if multigraph is connected and every vertex has an even degree. A multigraph satisfying the conditions for the existence the Eulerian circuit is called Eulerian multigraph. If the original multigraph is not Eulerian, then for UCPP solution some edges must be traversed more than once. In other words, the multigraph should be supplemented with copies of some the edges to the Eulerian multigraph, so that the cost of the added copies of the edges is minimal [4].

\subsection{The Directed Rural Chinese Postman problem}

The Directed Rural Chinese Postman Problem (DRCPP) is a special case of the WRCPP where a subset of the set of arcs of a given directed graph is required to be traversed at minimum cost $[2,8]$.

DRCPP is a special case of WRCPP, where $E=\varnothing$

In general, the DRCPP is NP-hard for directed multigraphs.

This problem also known as the Selecting Chinese Postman problem [8].

\subsection{The Directed Chinese Postman problem}

The Chinese Postman problem in the directed graph (Directed Chinese Postman Problem, DCPP) is a special case of the WRCPP problem, in which defined on directed graph and all arcs should be traversed. In some articles DCPP also called New York Street Sweeper Problem [8]

DCPP is a special case of WRCPP, where $E=\emptyset, A_{R}=A$

If multigraph has Eulerian trail, then this trail is a solution of DCPP. The algorithm for constructing the Eulerian trail has $O(|A|)$ time complexity [9].

The Eulerian trail is existing in a directed multigraph if multigraph is strongly connected and outdegree of each vertex is equal to indegree. A multigraph satisfying the conditions for the existence the Eulerian trail is called Eulerian multigraph. If the original multigraph is not Eulerian, then for DCPP solution some edges must be traversed more than once. In other words, the multigraph should be supplemented with copies of some the arcs to the Eulerian multigraph, so that the cost of the added copies of the arcs is minimal [2].

\subsection{The Undirected Windy Rural Chinese Postman problem}

The Undirected Windy Rural Chinese Postman Problem (UWRCPP) is an important ARP which generalizes most of the single-vehicle ARP and can be defined as follows $[2,9]$.

UWRCPP is a special case of WRCPP, where $A=\emptyset$ and there is edges, which satisfy (1).

\subsection{The Undirected Windy Chinese Postman problem}

The Undirected Windy Chinese Postman problem is the NP- hard problem of finding the minimum cost of a tour traversing all edges of an undirected multigraph, where the cost of traversal of an edge depends on the direction [10]

UWCPP is a special case of WRCPP, where $A=\emptyset$ and there is not edges, which satisfy (1). So, $\forall\left\{v_{i}, v_{j}\right\} \in E, C\left(v_{i}, v_{j}\right)=C\left(v_{j}, v_{i}\right)$.

224 
If multigraph has Eulerian circuit then this cycle is a solution of WCPP. The algorithm for constructing the Eulerian circuit has $O(|E|)$ time complexity [5]. If the original multigraph is not Eulerian, then some should be traversed more than once. In other words, the multigraph should be supplemented with copies of the edges to the Eulerian multigraph so that the cost of the added copies of the edges is minimal. The solution of the complement problem for a graph that does not satisfy properties (9) and (10) is an NP-hard problem. Thus, WCPP belongs to the class of NP- hard that cannot be solved in polynomial time [13].

\subsection{The Mixed Chinese Postman problem}

Mixed Chinese Postman Problem (MCPP) it is a version of WRCPP, where multigraph consists from edges and arcs, simultaneously, and all of them should be traversed $[11,12]$.

MCPP is a special case of WRCPP, where $A_{R}=A, E_{R}=E$. and there is not edges, which satisfy (1). So, $\forall\left\{v_{i}, v_{j}\right\} \in E, C\left(v_{i}, v_{j}\right)=C\left(v_{j}, v_{i}\right)$.

In 1962, Ford and Fulkerson proposed necessary and sufficient conditions for a mixed graph to be Eulerian. It is necessary and sufficient that in a strongly connected multigraph, the degrees of all vertices are even, and the divergence of each vertex is zero. If a mixed multigraph does not satisfy these conditions, then it must be supplemented by copies of arcs and edges to the Eulerian multigraph, so that the cost of the added copies of the arcs and edges is minimal. The addition of a mixed multigraph to Eulerian is an NP-difficult problem [13].

\subsection{The Mixed Windy Chinese Postman Problem}

The Mixed Windy Chinese Postman Problem (MWCCP, also called WCPP) is a special case of WRCPP. In MWCCP the cost of traversing the edges is depended from the direction of traversing.

UWRCPP is a special case of WRCPP, where there are edges, which satisfy (1).

In many theoretical works it was shown that problem is NP- hard.

\subsection{The Mixed Windy Chinese Postman Problem}

The Mixed Rural Chinese Postman Problem (MRCCP) is a special case of WRCPP In MRCCP not all edges and arcs should be traversed. There is a set of arcs and edges, which must appear in solution, other arcs and edges may appear in solution or may not.

MRCPP is a special case of WRCPP, where there are not edges, which satisfy (1)

In many theoretical works it was shown that problem is NP- hard [14].

We tried to build a classification of different CPP. Combine the existing CPP in a table containing the following criteria:

- the presence of set of edges (A)

- the presence of set of required edges (B),

- the presence of edges with cost, depending on traversing

- $\operatorname{direction}(\mathrm{C})$,

- the presence of set of arcs (D),

- the presence of set of required arcs (E).

The results are shown in "Table 1". As we can see, there are four problems, which today are not existing (yellow cells in table), but also can have real-world applications.

Table 1. The Classification of CPP

\begin{tabular}{|c|c|c|c|c|c|c|c|c|c|c|c|c|c|c|}
\hline & $\stackrel{0}{0}$ & 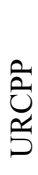 & $\begin{array}{l}\text { Uे } \\
\vdots \\
\vdots\end{array}$ & 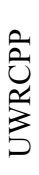 & 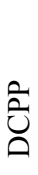 & $\begin{array}{l}\text { 今े } \\
\text { 苟 }\end{array}$ & $\hat{\text { ŝt }}$ & 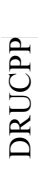 & $\begin{array}{l}\hat{0} \\
\dot{z} \\
\sum\end{array}$ & 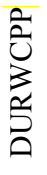 & 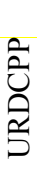 & $\begin{array}{l}\hat{\Delta} \\
\tilde{U} \\
\stackrel{\Sigma}{\Sigma}\end{array}$ & 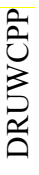 & $\frac{\hat{O}}{\sum_{0}^{0}}$ \\
\hline $\mathrm{A}$ & - & - & - & - & + & + & + & + & + & + & + & + & + & + \\
\hline $\mathrm{B}$ & - & - & - & - & - & + & - & - & - & - & + & + & + & + \\
\hline $\mathrm{C}$ & - & + & - & + & - & - & - & + & - & + & - & + & - & + \\
\hline $\mathrm{D}$ & + & + & + & + & - & - & + & + & + & + & + & + & + & + \\
\hline $\mathrm{E}$ & - & - & + & + & - & - & - & - & + & + & - & - & + & + \\
\hline
\end{tabular}

\section{Solving the Variations of Chinese Postman Problem}

In many sources was shown that almost all ARP problems can be transformed into VRP problems, predominantly in generalized travelling salesman problems (GTSP) $[13,15,16,17]$. For example, in [16] paper is described how the Capacitated Arc Routing Problem can be formulated as a standard vehicle routing problem. This allows us to transform arc routing into node routing problems and, therefore, establishes the equivalence of these two classes of problems. A well-known transformation by Pearn, Assad and Golden [16] reduces arc routing problem (ARP) into an equivalent vehicle routing problem (VRP). However, that transformation is regarded as unpractical, since an original instance with $n$ required edges is turned into a VRP over a complete graph with $3 n+1$ vertices. In [15] article was proposed a similar transformation that reduces this graph to $2 n+1$ vertices, with the additional restriction that a previously known set of $n$ pairwise disconnected edges must belong to every solution.

Thus, one can move from less studied problems ARP to well-known problems VRP, such as TSP and GTSP, which have a lot of different approximation algorithms for solving.

In the next sections, we try to compare the simplest algorithms for solving the GTSP. Generalized travelling salesman problem (GTSP) is an expansion of well-known TSP (Travelling Salesman Problem). In GTSP all vertices of graph are grouped in separate clusters. The solution of GTSP is a minimum-cost route, which traverse each cluster exactly once. 


\section{Methods for Solving the Generalized Travelling Salesman}

\section{Problem}

Now, we investigate the following simple approximate algorithms for solving GTSP:

- Nearest Neighbor Heuristic (NN) [19]

- Repetitive Nearest Neighbor Heuristic (RNN) [20];

- Improved Nearest Neighbor Heuristic (INN) [21];

- Repetitive Improved Nearest Neighbor Heuristic (RINN) [22];

- Loneliest Neighbor Heuristic (NLN) [23].

To evaluate the developed algorithms, the source code was written in the $\mathrm{C}++$ language.

Experiments was conducted on Apple Macbook Pro 13 a1502. Measurements were made of the executing time of the algorithm and the error rate of the solution. The results is presented in Table $1,2,3,4,5$ and $6 \cdot \operatorname{Min}(T), \max (T)$ and $M(T)$ means minimum, maximum and average time of algorithm working. $\operatorname{Min}(C), \max (C)$ and $M(C)$ means minimum, maximum and average error rate of algorithms.

Table 2. The measurements of NN algorithms

\begin{tabular}{|c|c|c|c|c|c|c|}
\hline$|\mathrm{V}|$ & $\min (T)$ & $\max (T)$ & $M(T)$ & $\min (C)$ & $\max (C)$ & $M(C)$ \\
\hline 50 & 0,001 & 0,079 & 0,003 & $6,65 \%$ & $23,53 \%$ & $14,59 \%$ \\
\hline 100 & 0,001 & 0,009 & 0,002 & $7,35 \%$ & $21,25 \%$ & $15,00 \%$ \\
\hline 200 & 0,002 & 0,025 & 0,004 & $8,93 \%$ & $21,77 \%$ & $15,24 \%$ \\
\hline 500 & 0,007 & 0,041 & 0,017 & $12,18 \%$ & $35,04 \%$ & $20,42 \%$ \\
\hline 1000 & 0,025 & 0,114 & 0,062 & $12,99 \%$ & $40,77 \%$ & $21,33 \%$ \\
\hline 1500 & 0,054 & 0,264 & 0,132 & $12,90 \%$ & $37,38 \%$ & $20,52 \%$ \\
\hline 2000 & 0,098 & 3,317 & 0,445 & $12,28 \%$ & $39,08 \%$ & $20,41 \%$ \\
\hline 3000 & 0,350 & 3,888 & 2,510 & $12,81 \%$ & $42,00 \%$ & $21,29 \%$ \\
\hline
\end{tabular}

Table 3. The measurements of RNN algorithms

\begin{tabular}{|c|c|c|c|c|c|c|}
\hline$|\mathrm{V}|$ & $\min (T)$ & $\max (T)$ & $M(T)$ & $\min (C)$ & $\max (C)$ & $M(C)$ \\
\hline 50 & 0,011 & 0,088 & 0,033 & $4,31 \%$ & $16,66 \%$ & $9,84 \%$ \\
\hline 100 & 0,079 & 2,521 & 0,261 & $6,07 \%$ & $15,52 \%$ & $11,07 \%$ \\
\hline 200 & 0,630 & 3,365 & 1,504 & $6,72 \%$ & $18,41 \%$ & $12,51 \%$ \\
\hline 500 & 6,678 & 98,085 & 31,772 & $10,08 \%$ & $30,40 \%$ & $17,83 \%$ \\
\hline 1000 & 62,258 & 695,821 & 247,218 & $11,65 \%$ & $37,44 \%$ & $18,51 \%$ \\
\hline 1500 & 198,014 & 2009,900 & 763,293 & $11,40 \%$ & $34,29 \%$ & $18,68 \%$ \\
\hline 2000 & 489,153 & 6731,020 & 2224,092 & $11,29 \%$ & $33,46 \%$ & $18,67 \%$ \\
\hline 3000 & 4102,820 & 8901,420 & 6334,230 & $12,53 \%$ & $38,94 \%$ & $20,59 \%$ \\
\hline
\end{tabular}

Table 4. The measurements of INN algorithms

\begin{tabular}{|c|c|c|c|c|c|c|}
\hline$|\mathrm{V}|$ & $\min (T)$ & $\max (T)$ & $M(T)$ & $\min (C)$ & $\max (C)$ & $M(C)$ \\
\hline 50 & 0,000 & 0,008 & 0,001 & $5,81 \%$ & $25,32 \%$ & $14,50 \%$ \\
\hline 100 & 0,000 & 0,009 & 0,001 & $7,48 \%$ & $22,43 \%$ & $14,79 \%$ \\
\hline 200 & 0,002 & 0,009 & 0,004 & $8,43 \%$ & $22,36 \%$ & $15,27 \%$ \\
\hline 500 & 0,011 & 0,054 & 0,025 & $12,57 \%$ & $35,96 \%$ & $20,30 \%$ \\
\hline 1000 & 0,040 & 0,178 & 0,095 & $12,64 \%$ & $40,29 \%$ & $20,63 \%$ \\
\hline 1500 & 0,091 & 0,395 & 0,207 & $12,24 \%$ & $38,45 \%$ & $20,31 \%$ \\
\hline 2000 & 0,155 & 1,035 & 0,404 & $12,09 \%$ & $35,56 \%$ & $20,24 \%$ \\
\hline 3000 & 0,369 & 21,215 & 4,478 & $12,65 \%$ & $42,12 \%$ & $21,20 \%$ \\
\hline
\end{tabular}

Table 5. The measurements of RINN algorithms

\begin{tabular}{|c|c|c|c|c|c|c|}
\hline$|\mathrm{V}|$ & $\min (T)$ & $\max (T)$ & $M(T)$ & $\min (C)$ & $\max (C)$ & $M(C)$ \\
\hline 50 & 0,001 & 0,029 & 0,008 & $6,49 \%$ & $22,54 \%$ & $14,07 \%$ \\
\hline 100 & 0,004 & 0,652 & 0,067 & $7,17 \%$ & $20,65 \%$ & $14,86 \%$ \\
\hline 200 & 0,050 & 1,212 & 0,372 & $8,65 \%$ & $23,28 \%$ & $15,14 \%$ \\
\hline 500 & 0,018 & 0,178 & 0,072 & $12,57 \%$ & $35,96 \%$ & $20,30 \%$ \\
\hline 1000 & 0,094 & 1,177 & 0,446 & $12,64 \%$ & $40,29 \%$ & $20,63 \%$ \\
\hline 1500 & 0,212 & 6,003 & 1,769 & $12,24 \%$ & $38,45 \%$ & $20,36 \%$ \\
\hline 2000 & 0,556 & 28,860 & 6,816 & $12,09 \%$ & $35,56 \%$ & $20,24 \%$ \\
\hline 3000 & 1,007 & 114,590 & 28,321 & $12,65 \%$ & $42,12 \%$ & $21,20 \%$ \\
\hline
\end{tabular}

Table 6. The measurements of NLN algorithms

\begin{tabular}{|c|c|c|c|c|c|c|}
\hline$|\mathrm{V}|$ & $\min (T)$ & $\max (T)$ & $M(T)$ & $\min (C)$ & $\max (C)$ & $M(C)$ \\
\hline 50 & 0,001 & 0,029 & 0,003 & $6,38 \%$ & $22,21 \%$ & $14,44 \%$ \\
\hline 100 & 0,004 & 0,046 & 0,011 & $8,03 \%$ & $20,63 \%$ & $14,76 \%$ \\
\hline 200 & 0,017 & 0,078 & 0,036 & $8,45 \%$ & $21,76 \%$ & $15,41 \%$ \\
\hline 500 & 0,078 & 0,434 & 0,190 & $12,52 \%$ & $34,46 \%$ & $20,36 \%$ \\
\hline 1000 & 0,293 & 2,262 & 0,814 & $12,65 \%$ & $40,48 \%$ & $20,68 \%$ \\
\hline 1500 & 0,656 & 15,192 & 3,043 & $12,81 \%$ & $36,84 \%$ & $20,17 \%$ \\
\hline 2000 & 0,356 & 19,953 & 3,764 & $12,59 \%$ & $38,07 \%$ & $21,32 \%$ \\
\hline 3000 & 1,456 & 120,110 & 27,402 & $13,34 \%$ & $39,91 \%$ & $22,33 \%$ \\
\hline
\end{tabular}

\section{Summary}

This article provides an overview of the known CPP. An attempt to systematize and classify these problems has been made. Mathematical formulations of new types of CPP was founded. The paper also shows that almost all problems of the ARP can be transformed to VRP. In addition, for solving the Chinese Postman problems the way of transformation it into VRP (mainly in GTSP) has been chosen.

228 
At this stage, the research is not complete. It is necessary to investigate the various ways of transformation ARP is into VRP. In addition, it is necessary to investigate the various ways of solving the GTSP. And the key idea of future research is the use of transformation algorithms and algorithms for solving the GTSP for solving the different modifications of CPP.

\section{References}

[1]. Eglese R., Letchford A., General Routing Problem. In Encyclopedia of Optimization. Springer, Boston, MA. 2008

[2]. Thimbleby, H. The directed chinese postman problem. Software: Practice and Experience, 33(11), 2003, pp. 1081-1096

[3]. Toth P., Vigo D. (ed.). The vehicle routing problem. - Society for Industrial and Applied Mathematics, 2002

[4]. Hertz A., Laporte G., Mittaz M. A tabu search heuristic for the capacitated arc routing problem. Operations research, vol. 48, no. 1, 2000, pp. 129-135.

[5]. Zerbino D. R., Birney E. Velvet: algorithms for de novo short read assembly using de Bruijn graphs. Genome research, vol. 18, no. 5, 2008, pp. 821-829.

[6]. Edmonds J., Johnson E. L. Matching, Euler tours and the Chinese postman. Mathematical programming, vol. 5, no. 1, 1973, pp. 88- 124.

[7]. Kolmogorov V. Blossom V: a new implementation of a minimum cost perfect matching algorithm. Mathematical Programming Computation, vol. 1, no. 1, 2009, pp. 43-67.

[8]. Robinson H. Graph theory techniques in model-based testing. In Proc. of the International Conference on Testing Computer Software, 1999.

[9]. Wilson R. J. An eulerian trail through Königsberg. Journal of graph theory, vol., 10, no. 3, 1986, pp. 265-275.

[10]. Ababei C., Kavasseri R. Efficient network reconfiguration using minimum cost maximum flow-based branch exchanges and random walks-based loss estimations, IEEE Transactions on Power Systems, vol. 26, no. 1, 2010, pp.. 30-37.

[11]. Chen W. H. Test sequence generation from the protocol data portion based on the Selecting Chinese Postman algorithm. Information Processing Letters, vol. 65, no. 5, pp. 261-268.

[12]. Aho A.V. et al. An optimization technique for protocol conformance test generation based on UIO sequences and rural Chinese postman tours. IEEE transactions on communications, vol. 39, no. 11, 1991, pp, 1604-1615.

[13]. Dror M. (ed.). Arc routing: theory, solutions and applications. Springer Science \& Business Media, 2012.

[14]. Ghiani G., Improta G. An algorithm for the hierarchical Chinese postman problem. Operations Research Letters, vol. 26, no. 1, 2000, pp. $27-32$.

[15]. Longo H., De Aragão M. P., Uchoa E. Solving capacitated arc routing problems using a transformation to the CVRP. Computers \& Operations Research, vol. 33, no. 6, pp. 18231837.

[16]. Pearn W. L., Assad A., Golden B. L. Transforming arc routing into node routing problems, Computers \& operations research, vol. 14, no. 4, 1987, pp. 285-288.

[17]. Laporte G. Modeling and solving several classes of arc routing problems as traveling salesman problems. Computers \& operations research, vol. 24, no. 11, 1997, pp. 10571061
[18]. Fischetti M., Salazar González J. J., Toth P. A branch-and-cut algorithm for the symmetric generalized traveling salesman problem. Operations Research, vol. 45, no. 3. 1997. pp. 378-394.

[19]. Solomon M. M. Algorithms for the vehicle routing and scheduling problems with time window constraints, Operations research, vol. 35, no. 2, 1987, pp. 254-265.

[20]. Modares A., Somhom S., Enkawa T. A self-organizing neural network approach for multiple traveling salesman and vehicle routing problems. International Transactions in Operational Research, vol. 6, no. 6, 1999, pp. 591-606.

[21]. Cheung K.L., Fu A.W.C. Enhanced nearest neighbor search on the R-tree. ACM SIGMOD Record, vol. 27, no. 3, 1998. pp. 16-21.

[22]. Tao Y., Papadias D., Shen Q. Continuous nearest neighbor search. In Proceedings of the 28th International Conference on Very Large Databases, 2002, pp. 287-298.

[23]. Pimentel F. G. S. L. Double-ended nearest and loneliest neighbour: a nearest neighbour heuristic variation for the travelling salesman problem. Revista de Ciências da Computação, vol. 6, issue 6, 2011.

\section{Варианты задач китайского почтальона и их решения через преобразование в задачи маршрутизации}

\author{
M.K. Горденко <mgordenko@hse.ru> \\ C.M. Авдошин <savdoshin@hse.ru> \\ Департамент программной инженерии \\ Национальный исследовательский университет “Высшая школа экономики”,
} 101000, Россия, г. Москва, ул. Мясниикая, д. 20

Abstract. В статье описаны проблемы маршрутизации. Показано, что почти все проблемы маршрутизации дуг могут быть преобразованы в другие проблемы маршрутизации. Это продемонстрировано на примере задачи китайского почтальона в смешанном мультиграфе. Также в статье приведен обзор различных задач китайского почтальона (в зависимости от типа графа, функции стоимости и обязательности прохождения элементов графа). Для каждой проблемы дана математическая постановка Кроме того, описаны примеры потенциально полезных приложений, где задачи могут быть применены. Приведена таблица различных вариантов задачи китайского почтальона и выбраны параметры для идентификации различных типов задач. Выделено пять параметров: наличие дуг, наличие ребер, наличие обязательных дуг, наличие обязательных ребер, наличие ребер со стоимостью, зависящей от прохода. Показано, что, варьируя эти параметры, можно получить новые задачи, которые могут быть полезны в реальной жизни, однако еще не описаны. Выявлены четыре таких задачи. Показано, что задача китайского почтальона может быть решена путем преобразования в другие задачи маршрутизации. Приведен метод, позволяющий преобразовать задачу в обобщенную задачу коммивояжера. Показаны результаты применения простейших алгоритмов для решения преобразованного варианта задачи (результаты приведены для алгоритмов ближайшего соседа и их модификаций). Исследование еще не завершено, планируется продолжать тестировать алгоритмы решения смежных задач маршрутизации и алгоритмы для преобразований задач в эквивалентные. 
Keywords: обобщенная задача коммивояжера; задача маршрутизации дуг; задача маршрутизации; обобщенная задача маршрутизации; задача китайского почтальона

DOI: $10.15514 /$ ISPRAS-2018-30(3)-16

Для цитирования: Горденко М.К., Авдошин С.М. Варианты задач китайского почтальона и их решения через преобразование в задачи маршрутизации. Труды ИСП PAH, том 30, вып. 3, 2018 г., стр. 221-232 (на английском языке). DOI: 10.15514/ISPRAS2018-30(3)-16

\section{Список литературы:}

11]. Eglese R., Letchford A., General Routing Problem. In Encyclopedia of Optimization. Springer, Boston, MA. 2008

[2]. Thimbleby, H. The directed chinese postman problem. Software: Practice and Experience, 33(11), 2003, pp. 1081-1096.

[3]. Toth P., Vigo D. (ed.). The vehicle routing problem. - Society for Industrial and Applied Mathematics, 2002

[4]. Hertz A., Laporte G., Mittaz M. A tabu search heuristic for the capacitated arc routing problem. Operations research, vol. 48, no. 1, 2000, pp. 129-135.

[5]. Zerbino D. R., Birney E. Velvet: algorithms for de novo short read assembly using de Bruijn graphs. Genome research, vol. 18, no. 5, 2008, pp. 821-829.

[6]. Edmonds J., Johnson E. L. Matching, Euler tours and the Chinese postman. Mathematical programming, vol. 5, no. 1, 1973, pp. 88- 124.

[7]. Kolmogorov V. Blossom V: a new implementation of a minimum cost perfect matching algorithm. Mathematical Programming Computation, vol. 1, no. 1, 2009, pp. 43-67.

[8]. Robinson H. Graph theory techniques in model-based testing. In Proc. of the International Conference on Testing Computer Software, 1999.

[9]. Wilson R. J. An eulerian trail through Königsberg. Journal of graph theory, vol., 10, no. 3, 1986, pp. 265-275.

[10]. Ababei C., Kavasseri R. Efficient network reconfiguration using minimum cost maximum flow-based branch exchanges and random walks-based loss estimations, IEEE Transactions on Power Systems, vol. 26, no. 1, 2010, pp.. 30-37.

[11]. Chen W. H. Test sequence generation from the protocol data portion based on the Selecting Chinese Postman algorithm. Information Processing Letters, vol. 65, no. 5, pp. 261-268.

[12]. Aho A.V. et al. An optimization technique for protocol conformance test generation based on UIO sequences and rural Chinese postman tours. IEEE transactions on communications, vol. 39, no. 11, 1991, pp, 1604-1615.

[13]. Dror M. (ed.). Arc routing: theory, solutions and applications. Springer Science \& Business Media, 2012.

[14]. Ghiani G., Improta G. An algorithm for the hierarchical Chinese postman problem. Operations Research Letters, vol. 26, no. 1, 2000, pp. $27-32$.

[15]. Longo H., De Aragão M. P., Uchoa E. Solving capacitated arc routing problems using a transformation to the CVRP. Computers \& Operations Research, vol. 33, no. 6, pp. 18231837.

[16]. Pearn W. L., Assad A., Golden B. L. Transforming arc routing into node routing problems, Computers \& operations research, vol. 14, no. 4, 1987, pp. 285-288.
117]. Laporte G. Modeling and solving several classes of arc routing problems as traveling salesman problems. Computers \& operations research, vol. 24, no. 11, 1997, pp. 1057 1061.

[18]. Fischetti M., Salazar González J. J., Toth P. A branch-and-cut algorithm for the symmetric generalized traveling salesman problem. Operations Research, vol. 45, no. 3. 1997. pp. 378-394.

[19]. Solomon M. M. Algorithms for the vehicle routing and scheduling problems with time window constraints, Operations research, vol. 35, no. 2, 1987, pp. 254-265.

[20]. Modares A., Somhom S., Enkawa T. A self-organizing neural network approach for multiple traveling salesman and vehicle routing problems. International Transactions in Operational Research, vol. 6, no. 6, 1999, pp. 591-606.

[21]. Cheung K.L., Fu A.W.C. Enhanced nearest neighbor search on the R-tree. ACM SIGMOD Record, vol. 27, no. 3, 1998. pp. 16-21.

[22]. Tao Y., Papadias D., Shen Q. Continuous nearest neighbor search. In Proceedings of the 28th International Conference on Very Large Databases, 2002, pp. 287-298.

[23]. Pimentel F. G. S. L. Double-ended nearest and loneliest neighbour: a nearest neighbour heuristic variation for the travelling salesman problem. Revista de Ciências da Computação, vol. 6, issue 6,2011 . 